\title{
Bone Loss, Osteoporosis, and Fractures in Patients with Rheumatoid Arthritis: A Review
}

\author{
Patrice Fardellone $^{1, *}$, Emad Salawati ${ }^{2}$ (I) , Laure Le Monnier ${ }^{1}$ and Vincent Goëb ${ }^{1}(\mathbb{C}$ \\ 1 Department of Rheumatology, Picardie-Jules Verne University, University hospital of Amiens, 80054 Amiens, \\ France; LeMonnier.Laure@chu-amiens.fr (L.L.M.); goeb.vincent@chu-amiens.fr (V.G.) \\ 2 Assistant Professor, Faculty of Medicine, King Abdulaziz University, 21589 Jeddah, Saudi Arabia; \\ esalawati@kau.edu.sa \\ * Correspondence: fardellone.patrice@chu-amiens.fr
}

Received: 28 August 2020; Accepted: 15 October 2020; Published: 20 October 2020

\begin{abstract}
Rheumatoid arthritis (RA) is often characterized by bone loss and fragility fractures and is a frequent comorbidity. Compared with a matched population, RA patients with fractures have more common risk factors of osteoporosis and fragility fractures but also risk factors resulting from the disease itself such as duration, intensity of the inflammation and disability, and cachexia. The inflammatory reaction in the synovium results in the production of numerous cytokines (interleukin-1, interleukin-6, tumor necrosis factor) that activate osteoclasts and mediate cartilage and bone destruction of the joints, but also have a systemic effect leading to generalized bone loss. Regular bone mineral density (BMD) measurement, fracture risk assessment using tools such as the FRAX algorithm, and vertebral fracture assessment (VFA) should be performed for early detection of osteoporosis and accurate treatment in RA patients.
\end{abstract}

Keywords: rheumatoid arthritis; osteoporosis; fragility fractures; BMD; bone remodeling markers

\section{Introduction}

Rheumatoid arthritis is the most common immune disease of the skeleton. During the last three decades, many studies have shown that besides the joint inflammation and destruction, bone mass in patients with rheumatoid arthritis (RA) is lower than in the matched non-RA population [1-9] and the risk of osteoporosis is increased [8,10-19]. Similarly, the incidence of osteoporotic fractures is high $[6,11,12,14-25]$ even when compared to the general population of the same age and $\operatorname{sex}[5,9,15,26-42]$. Osteoporosis-related fragility fractures represent one of the most severe complications in RA patients [43]. They contribute to a significant decrease in the quality of life and can reduce life expectancy. In the United States, data from the National Data Bank for Rheumatic Diseases indicated that osteoporotic fractures are the third cause of mortality in RA patients [44]. The severe inflammatory reaction in the synovium results in the production of numerous cytokines (interleukin-1, interleukin-6, tumor necrosis factor) that mediate cartilage and bone destruction but also have a systemic effect leading to generalized bone loss. As in the general population, the highly sensitive C-reactive protein (CRP) level is a predictor of the risk of fracture that is underlying the role of systemic inflammation [45]. The mechanism of bone loss relies on increased bone resorption mediated by pro-inflammatory cytokines that activate osteoclasts, as well as by the frequent use of glucocorticoids or inactivity resulting from the disease. Some clues lowering the level of inflammation with potent drugs such as biologic agents can reduce the risk of osteoporosis and fractures. The literature provides interesting data on the prevalence of osteoporosis in RA patients, as well as the incidence of fragility fractures compared to a healthy population. 


\section{Epidemiology of Bone Loss and Fractures in Rheumatoid Patients}

\subsection{Low Bmd}

According to the definition given by the WHO organization in 1994, based on a bone mineral density (BMD) T-score $\leq 2.5$ SD measured by dual-energy X-ray absorptiometry (DEXA) in the spine and/or hip, it is possible to evaluate the prevalence of osteoporosis in the RA population. In this population, decreased mean BMD values of the spine and hips are known to be a very common feature and are more frequent than in a paired healthy control population (Table 1). A significant bone loss appears early in RA. After a two-year duration of the disease, the hip and spine BMD show an already significant loss in all locations, with a significantly greater BMD loss in the hands (BMD measured in metacarpals $2-4$ by digital x-ray radiogrammetry) than a generalized BMD loss in the hip and spine [46]. In an established RA, observational and case-control studies estimate the prevalence of loss of BMD to be doubled in both male and female RA patients compared with healthy controls at both the lumbar spine and the hip (Tables 1 and 2). Results are expressed whether in T-score or Z-score. T-score is a young gender-matched population at peak bone mass, while the Z-score is derived from an age-matched reference population. Both T-scores and Z-scores are derived by comparison to the reference population on a standard deviation scale.

As shown in Table 1, among the classical risk factors of bone loss, glucocorticoids (GCs) commonly prescribed in RA patients are also well known for causing secondary osteoporosis with the long use of high dosage when comparing users with non-users [1,2,4]. Inversely, it seems that at low dosage, glucocorticoids exert a protective effect on the bone probably because the lowering of the inflammation is more beneficial than the deleterious effects of the drug [47].

\subsection{Fragility Fractures}

Epidemiological studies on the frequency of fractures in RA populations can be observational, case-controlled, conducted on databases, or prospective (Tables 3 and 4). Most epidemiological studies provide a fractures risk increased by 1.5- to 2-fold among patients with RA compared to the general population (Table 4).

Patients with RA are at a high risk of vertebral fractures. However, the prevalence of spine fractures varies enormously according to the population studied (age, women alone, or men and women), the way to assess fractures (radiographs or vertebral fracture assessment (VFA) for vertebral fractures), and the source of data (cohort, registry, randomized trial). This explains that the prevalence of vertebral fractures ranges from $8 \%$ to nearly $50 \%$ (Table 3 ).

On the other hand, the risk of fractures, despite the site, is always higher in RA patients than in the general population (Table 4). In a very large case-control study of 30,262 patients with RA using the British General Practice Research Database, the increased risk of fracture compared to the general population was most marked at the hip (RR: 2.0, 95\% CI 1.8-2.3) and spine (RR: $2.4,95 \%$ CI 2.0-2.8) [32]. The relationship between RA and the fracture was examined in the Women's Health Initiative prospective study (WHI). Compared to the non-arthritis group, the risk (HR $(95 \% \mathrm{CI})$ ) of sustaining any clinical fracture comprising self-reported spinal fractures in the RA group was 1.49 $(1.26,1.75)(p<0.001)$. Moreover, the risk of sustaining a hip fracture significantly increased in the RA group $(3.03(2.03,4.51))(p<0.001)$ [34]. 
Table 1. Bone mineral density (BMD) in rheumatoid arthritis (RA) patients: Observational studies.

\begin{tabular}{|c|c|c|c|c|c|}
\hline \multirow{3}{*}{$\begin{array}{l}\text { Authors } \\
\text { Country } \\
\text { Year }\end{array}$} & \multirow{3}{*}{ Studied Population } & \multirow{3}{*}{ Type of the Study } & \multicolumn{3}{|c|}{ BMD } \\
\hline & & & \multicolumn{3}{|c|}{ Sites } \\
\hline & & & Total Hip & Femoral Neck & Lumbar Spine \\
\hline $\begin{array}{l}\text { Laan RFJM } \\
\text { The Netherlands } \\
1993 \text { [10] }\end{array}$ & $\begin{array}{c}\text { men and women }(58.7 \%) \\
\text { mean age: } \\
\text { women } 57.595 \% \text { CI } 27 \text { to } 78 \\
\text { men } 5795 \% \text { CI } 30 \text { to } 80\end{array}$ & $\begin{array}{l}\text { longitudinal } \\
\quad 97 \mathrm{RA}\end{array}$ & $\begin{array}{c}\text { Z-score }<-2=4.4 \% 95 \% \\
\text { CI } 0.2 \text { to } 8.6\end{array}$ & & $\begin{array}{c}\text { Z-score }<-2=9.3 \% 95 \% \\
\text { CI } 3.5 \text { to } 15.0\end{array}$ \\
\hline $\begin{array}{l}\text { Sinigaglia L } \\
\text { Italy } \\
2000[11] \\
\end{array}$ & $\begin{array}{c}\text { women } \\
20 \text { to } 70 \text { years }\end{array}$ & $\begin{array}{l}\text { observational } \\
\text { 925 RA }\end{array}$ & & $36.2 \%$ T-score $\leq-2.5 \mathrm{SD}$ & $28.8 \%$ T-score $\leq-2.5 \mathrm{SD}$ \\
\hline $\begin{array}{l}\text { Kvien TK } \\
\text { Norway } \\
2000[12]\end{array}$ & $\begin{array}{c}\text { women } \\
\text { mean age: } 54.8 \pm 11.6 \text { years } \\
\text { Oslo RA Register }\end{array}$ & $\begin{array}{l}\text { observational } \\
\text { 394 RA }\end{array}$ & $\begin{array}{c}14.7 \% \text { T-score } \leq-2.5 \mathrm{SD} \\
95 \% \text { CI } 11.1 \text { to } 18\end{array}$ & $14.7 \% 95 \%$ CI 11.1 to 18.3 & $16.8 \% 95 \%$ CI 13.1 to 20.5 \\
\hline $\begin{array}{l}\text { Haugeberg G } \\
\text { Norway } \\
2000[13]\end{array}$ & $\begin{array}{l}\text { women } \\
\text { age: } 20 \text { to } 70 \text { years } \\
\text { county RA register }\end{array}$ & $\begin{array}{l}\text { observational } \\
394 \text { RA }\end{array}$ & $\begin{array}{c}\text { all RA patients: } 14.7 \% \\
\text { T-score } \leq-2.5 \text { SD } \\
95 \% \text { CI } 11.1 \text { to } 18.3 \\
\text { corticoid non-users: } 5.8 \\
\text { T-score } \leq-2.5 \text { SD } \\
95 \% \text { CI } 1.9 \text { to } 9.7 \\
\text { corticoid users: } 24.0 \\
\text { T-score } \leq-2.5 \text { SD } \\
\text { 95\% CI } 17.1 \text { to } 30.9\end{array}$ & $\begin{array}{c}\text { all RA patients: } 14.7 \% \\
\text { T-score } \leq-2.5 \mathrm{SD} \\
95 \% \text { CI } 11.1 \text { to } 18.3 \\
\text { corticoid non-users: } 6.5 \\
\text { 95\% T-score } \leq-2.5 \mathrm{SD} \\
95 \% \text { CI } 2.4 \text { to } 10.6 \\
\text { corticoid users: } 24.7 \\
\text { T-score } \leq-2.5 \mathrm{SD} \\
95 \% \text { CI } 17.7 \text { to } 31.7\end{array}$ & $\begin{array}{c}\text { all RA patients: } 16.8 \% \\
\text { T-score } \leq-2.5 \text { SD } \\
95 \% \text { CI } 13.1 \text { to } 20.5 \\
\text { corticoid non-users: } 8.6 \% \\
\text { T-score } \leq-2.5 \text { SD } \\
95 \% \text { CI } 3.9 \text { to } 13.3 \\
\text { corticoid users: } 26.6 \% \\
\text { T-score } \leq-2.5 \text { SD } \\
\text { 95\% CI } 19.6 \text { to } 33.6\end{array}$ \\
\hline $\begin{array}{l}\text { Lodder MC } \\
\text { Norway, } \\
\text { UK, The } \\
\text { Netherlands } \\
2003 \text { [14] }\end{array}$ & $\begin{array}{l}\text { women from OSTRA cohort } \\
\text { mean age: } 61.0 \pm 5.8 \text { years } \\
\text { age: } 50 \text { to } 70 \text { years }\end{array}$ & $\begin{array}{l}\text { observational } \\
150 \text { RA }\end{array}$ & $8 \%$ T-score $\leq-2.5 \mathrm{SD}$ & $12 \%$ T-score $\leq-2.5 \mathrm{SD}$ & $10.7 \%$ T-score $\leq-2.5 \mathrm{SD}$ \\
\hline $\begin{array}{l}\text { Ørstavik RE } \\
\text { Norway } \\
2003[15]\end{array}$ & $\begin{array}{c}\text { women } \\
\text { Oslo RA register } \\
\text { mean age: } 63.4 \text { (51.4 to } 73.6) \text { years }\end{array}$ & $\begin{array}{l}\text { observational } \\
229 \text { RA }\end{array}$ & $16.6 \%$ T-score $\leq-2.5 \mathrm{SD}$ & $20.3 \%$ T-score $\leq-2.5 \mathrm{SD}$ & $21.8 \%$ T-score $\leq-2.5 \mathrm{SD}$ \\
\hline $\begin{array}{l}\text { El Maghraoui } \\
\text { Morocco } \\
2010[16] \\
\end{array}$ & $\begin{array}{c}\text { women } \\
\text { mean age: } 49.4 \pm 7.3 \text { years } \\
\text { age: } 27 \text { to } 70 \text { years }\end{array}$ & $\begin{array}{l}\text { observational } \\
\text { 172 RA }\end{array}$ & $\begin{array}{l}\text { Any site: } 44.2 \% \\
\text { T-score } \leq-2.5 \mathrm{SD}\end{array}$ & & \\
\hline $\begin{array}{l}\text { Lee SG } \\
\text { South Korea } \\
2012[8]\end{array}$ & $\begin{array}{c}\text { women } \\
\text { age: } 20 \text { to } 80 \text { years }\end{array}$ & $\begin{array}{c}\text { case-control } \\
\text { 299 RA } \\
246 \text { non-RA controls }\end{array}$ & $7.8 \%^{* *} \mathrm{~T}$-score $\leq-2.5 \mathrm{SD}$ & & $\begin{array}{c}18.2 \% \mathrm{~T} \text {-score } \leq-2.5 \mathrm{SD} \\
p=0.067\end{array}$ \\
\hline
\end{tabular}


Table 1. Cont

\begin{tabular}{|c|c|c|c|c|c|}
\hline $\begin{array}{l}\text { Brébant S } \\
\text { France } \\
2012[17]\end{array}$ & $\begin{array}{c}\text { women } \\
\text { mean age: } 56.0 \pm 13.5 \text { years }\end{array}$ & $\begin{array}{l}\text { observational } \\
\text { 185 RA }\end{array}$ & $21.2 \%$ T-score $\leq-2.5 \mathrm{SD}$ & $33.3 \%$ T-score $\leq-2.5 \mathrm{SD}$ & $24.2 \%$ T-score $\leq-2.5 \mathrm{SD}$ \\
\hline $\begin{array}{l}\text { El Maghraoui } \\
\text { Morocco } \\
2015 \text { [18] }\end{array}$ & $\begin{array}{c}\text { men and women }(82.6 \%) \\
\text { mean age: } 54.1 \pm 11.5 \text { years } \\
\text { age: } 25 \text { to } 82 \text { years }\end{array}$ & $\begin{array}{l}\text { observational } \\
\text { 178 RA }\end{array}$ & $\begin{array}{l}\text { overall population: } 29.2 \% \\
\text { T-score } \leq-2.5 \mathrm{SD}\end{array}$ & & \\
\hline $\begin{array}{c}\text { Choi ST } \\
\text { South Korea } \\
2018 \text { [19] }\end{array}$ & $\begin{array}{l}\text { men and women }(88.9 \%) \\
\text { mean age: } 61.8 \pm 11.5 \text { years }\end{array}$ & $\begin{array}{c}\text { retrospective } \\
\text { cross-sectional study } \\
479 \mathrm{RA}\end{array}$ & $\begin{array}{c}\text { overall population: } 91.3 \% \\
\text { T-score } \leq-2.5 \mathrm{SD} \\
\text { men: } 90 \% \text { T-score } \leq-2 \\
\text { T.5 SD } \\
\text { women: } 91.3 \% \\
\text { T-score } \leq-2.5 \mathrm{SD}\end{array}$ & & \\
\hline
\end{tabular}

Level of significance between RA and non-RA controls: ${ }^{* *} p<0.02$; NS: Non-significant; OR: Odd ratio; RR: Relative risk; HR: Hazard ratio.

Table 2. BMD in RA patients: Case-control studies.

\begin{tabular}{|c|c|c|c|c|c|}
\hline \multirow{3}{*}{$\begin{array}{l}\text { Authors } \\
\text { Country } \\
\text { Year } \\
\text { (Ref) }\end{array}$} & \multirow{3}{*}{ Studied Population } & \multirow{3}{*}{$\begin{array}{c}\text { Type of the Study } n \\
\text { RA/ } \\
n \text { Controls }\end{array}$} & \multicolumn{3}{|c|}{ BMD } \\
\hline & & & \multicolumn{3}{|c|}{ Sites } \\
\hline & & & Total Hip & Femoral Neck & Lumbar Spine \\
\hline $\begin{array}{c}\text { Garton MJ } \\
\text { UK } \\
1993[1]\end{array}$ & $\begin{array}{c}\text { men } \\
\text { age: } 47 \text { to } 74 \text { years }\end{array}$ & $\begin{array}{c}40 \text { RA } \\
20 \text { non-RA controls }\end{array}$ & $\begin{array}{c}\text { Trochanter } \\
\text { RA corticoid users vs. } \\
\text { non-RA: }-15.9 \%{ }^{* *} \\
\text { RA non-users vs. } \\
\text { non-RA: }-10.1^{*}\end{array}$ & & $\begin{array}{c}\text { RA corticoid users vs. } \\
\text { non-RA: }-12.0 \%{ }^{* *} \\
\text { RA non-users vs. non-RA: }-11.2 * *\end{array}$ \\
\hline $\begin{array}{c}\text { Hall GM } \\
\text { UK } \\
1993[2]\end{array}$ & postmenopausal women & $\begin{array}{c}195 \text { RA } \\
597 \text { non-RA controls }\end{array}$ & $\begin{array}{c}\text { RA corticoid users vs. } \\
\text { non-users: }-7.4^{* * *} \\
\text { 95\% CI }-1.2 \text { to } 13.6 \\
\text { RA corticoid non-users vs. } \\
\text { non-RA: }-6.9 \%{ }^{*} \\
\text { 95\% CI } 3.4 \text { to } 10.3 \\
\text { RA corticoid users vs. } \\
\text { non-RA: }-13.8 \% * \\
\text { 95\% CI } 8.6 \text { to } 19.0\end{array}$ & $\begin{array}{l}\text { RA corticoid users vs. RA } \\
\text { non-users: }-6.9^{* *} \\
95 \% \text { CI }-3.4 \text { to }-10.3\end{array}$ & $\begin{array}{c}\text { RA corticoid users vs. RA } \\
\text { non-users: }-6.5^{*} \\
95 \% \text { CI } 0 \text { to } 13.0 \\
\text { RA corticoid non-users vs. } \\
\text { non-RA: NS } \\
\text { RA corticoid users vs. non-RA: }-7.5 * \\
95 \% \text { CI } 1.8 \text { to } 13.2\end{array}$ \\
\hline
\end{tabular}


Table 2. Cont.

\begin{tabular}{|c|c|c|c|c|c|}
\hline $\begin{array}{c}\text { Gough AKS } \\
\text { UK } \\
1994[3]\end{array}$ & men and women $(66.9 \%)$ & $\begin{array}{c}148 \text { RA } \\
50 \text { non-RA controls }\end{array}$ & $\begin{array}{c}\text { loss of BMD in 1-year at } \\
\text { the trochanter } \\
\text { all: }-2.2 \pm 0.5^{* * *} \\
\text { male: }-0.6 \pm 0.6^{* * *} \\
\text { female: }-2.9 \pm 0.6^{* * *}\end{array}$ & $\begin{array}{l}\text { loss of BMD in 1-year } \\
\text { all: }-2.0 \pm 0.4 \\
\text { male: }-12 \pm 0.7 \\
\text { female: }-23 \pm 0.6\end{array}$ & $\begin{array}{l}\text { loss of BMD in 1-year } \\
\text { all: }-1.0 \pm 0.3^{*} \\
\text { male: }-0.2 \pm 0.5^{*} \\
\text { female: }-13 \pm 0.4^{*}\end{array}$ \\
\hline $\begin{array}{l}\text { Lane NE } \\
\text { USA } \\
1995[4]\end{array}$ & $\begin{array}{l}\text { non-black women } \geq 65 \text { years } \\
\text { SOF study }\end{array}$ & $\begin{array}{l}120 \text { RA } \\
7966 \text { non-RA } \\
\text { controls }\end{array}$ & $\begin{array}{c}\text { RA corticoid non-users vs. } \\
\text { non-RA: }-7.3^{*} \\
\text { 95\% CI }-11.4 \text { to }-3.2 \\
\text { RA corticoid users vs. } \\
\text { non-RA: }-14.7^{*} \\
95 \% \text { CI }-23.4 \text { to } 5.9\end{array}$ & & $\begin{array}{c}\text { RA corticoid non users vs. } \\
\text { non-RA: }-8.4^{*} \\
95 \% \text { CI }-13.4 \text { to }-3.4 \\
\text { RA corticoid users vs. non-RA: NS } \\
\text { (too small number) }\end{array}$ \\
\hline $\begin{array}{l}\text { Martin JC } \\
\text { UK } \\
1997[5]\end{array}$ & $\begin{array}{l}\text { caucasian postmenopausal women } \\
\text { from EVOS }\end{array}$ & $\begin{array}{c}46 \text { RA } \\
29 \text { non-RA controls }\end{array}$ & & RA vs. non-RA: $-15.4 \% * *$ & RA vs. non-RA: $-6.7 \%$ \\
\hline $\begin{array}{l}\text { Haugeberg G } \\
\text { Norway } \\
2000[7]\end{array}$ & $\begin{array}{c}\text { men } \\
\text { age: } 20 \text { to } 70 \text { years } \\
\text { county RA register }\end{array}$ & $\begin{array}{c}\text { case-control 94 RA } \\
\text { men } \\
1130 \text { non-RA } \\
\text { controls: } \\
\text { European/United } \\
\text { States reference } \\
\text { population } \\
\end{array}$ & $\begin{array}{l}\text { age group } 60-70 \\
\text { years: }-6.9 \% *\end{array}$ & $\begin{array}{l}\text { age group } 60-70 \\
\text { years: }-5.2 \% *\end{array}$ & NS \\
\hline $\begin{array}{l}\text { Ørstavik RE } \\
\text { Norway } \\
2004[6]\end{array}$ & $\begin{array}{c}\text { women } \\
\text { Oslo RA register } \\
\text { mean age: } 63.0(50.7 \text { to } 73.6) \text { years }\end{array}$ & 249 RA & $\begin{array}{l}17.3 \% \text { T-score } \leq-2.5 \mathrm{SD}^{*} \\
\mathrm{OR}=5.895 \% \mathrm{CI} 2.4 \text { to } 17.0\end{array}$ & $\begin{array}{l}18.6 \% \text { T-score } \leq-2.5 \mathrm{SD}^{*} \\
\mathrm{OR}=4.195 \% \mathrm{CI} 2.0 \text { to } 9.7\end{array}$ & $\begin{array}{l}22.0 \% \text { T-score } \leq-2.5 \mathrm{SD}^{*} \\
\mathrm{OR}=2.095 \% \mathrm{CI} 1.2 \text { to } 3.4\end{array}$ \\
\hline $\begin{array}{c}\text { Lee SG } \\
\text { South Korea } \\
2012[8]\end{array}$ & $\begin{array}{l}\text { women } \\
\text { age: } 20 \text { to } 80 \text { years }\end{array}$ & $\begin{array}{c}299 \text { RA } \\
246 \text { non-RA controls }\end{array}$ & $7.8 \%^{* *} \mathrm{~T}$-score $\leq-2.5 \mathrm{SD}$ & & $\begin{array}{c}18.2 \% \text { T-score } \leq-2.5 \mathrm{SD} \\
\quad p=0.067\end{array}$ \\
\hline $\begin{array}{l}\text { Avouac J } \\
\text { France } \\
2012[9]\end{array}$ & $\begin{array}{c}\text { women } \\
\text { mean age: } 61 \pm 11 \text { years }\end{array}$ & $\begin{array}{c}139 \text { RA } \\
227 \text { non-RA controls }\end{array}$ & $19 \%$ T-score $\leq-2.5 \mathrm{SD}^{* * *}$ & & $21 \%$ T-score $\leq-2.5 \mathrm{SD}^{* * *}$ \\
\hline
\end{tabular}

Level of significance between RA and non-RA controls: ${ }^{*} p<0.05 ;{ }^{* *} p<0.02 ;{ }^{* * *} p<0.001$; NS: Non-significant. 
Table 3. Incidence, prevalence, and risk of fractures in RA patients: Observational studies.

\begin{tabular}{|c|c|c|c|c|c|c|}
\hline \multirow{2}{*}{$\begin{array}{l}\text { Authors } \\
\text { Country } \\
\text { Year } \\
\text { (Ref) }\end{array}$} & \multirow[b]{2}{*}{ Studied Population } & \multirow[b]{2}{*}{$\begin{array}{c}\text { Type of Study } \\
n \text { RA }\end{array}$} & \multicolumn{4}{|c|}{ Fractures Sites } \\
\hline & & & Vertebral & $\begin{array}{l}\text { Proximal } \\
\text { Femur }\end{array}$ & Non-Vertebral & Any Site \\
\hline $\begin{array}{l}\text { Kvien TK } \\
\text { Norway } \\
2000[12]\end{array}$ & $\begin{array}{c}\text { women } \\
\text { age: } 54.8 \pm 11.6 \text { years } \\
\text { Oslo RA Register } \\
\end{array}$ & $\begin{array}{c}\text { observational } \\
394 \text { RA }\end{array}$ & & & $21.2 \%$ & \\
\hline $\begin{array}{l}\text { Sinigaglia L } \\
\text { Italy } \\
2000[11]\end{array}$ & $\begin{array}{c}\text { women } \\
20 \text { to } 70 \text { years }\end{array}$ & $\begin{array}{c}\text { observational } \\
925 \text { RA }\end{array}$ & $8.0 \%$ & & & \\
\hline $\begin{array}{c}\text { Lodder MC } \\
\text { Norway } \\
\text { UK, The Netherlands } \\
2003[14] \\
\end{array}$ & $\begin{array}{c}\text { women from OSTRA cohort } \\
\text { mean age: } 61.0 \pm 5.8 \text { years } \\
\text { age: } 50 \text { to } 70 \text { years }\end{array}$ & $\begin{array}{c}\text { observational } \\
150 \mathrm{RA}\end{array}$ & $16 \%$ & & & \\
\hline $\begin{array}{l}\text { Ørstavik RE } \\
\text { Norway } \\
2002[15]\end{array}$ & $\begin{array}{c}\text { women } \\
\text { Oslo RA register } \\
\text { mean age: } 63.4 \text { (51.4 to } 73.6) \text { years }\end{array}$ & $\begin{array}{c}\text { observational } \\
229 \text { RA }\end{array}$ & $48.9 \%$ & & & \\
\hline $\begin{array}{l}\text { Ørstavik RE } \\
\text { Norway } \\
2005 \text { [6] }\end{array}$ & $\begin{array}{c}\text { women } \\
\text { Oslo RA register } \\
\text { mean age: } 53.0 \pm 11.2 \text { years }\end{array}$ & $\begin{array}{l}\text { longitudinal } \\
255 \mathrm{RA}\end{array}$ & $\begin{array}{c}15.0 \%(\mathrm{VFA}) \\
\mathrm{IR}=2.9 / 100 \\
\text { person-years }\end{array}$ & & & \\
\hline $\begin{array}{l}\text { El Maghraoui } \\
\text { Morocco } \\
2010[16]\end{array}$ & $\begin{array}{c}\text { women } \\
\text { mean age: } 49.4 \pm 7.3 \text { years } \\
\text { age: } 27 \text { to } 70 \text { years }\end{array}$ & $\begin{array}{c}\text { observational } \\
172 \mathrm{RA}\end{array}$ & $36 \%$ (VFA) & & & \\
\hline $\begin{array}{c}\text { Vis M } \\
\text { Norway, } \\
\text { UK, The Netherlands } \\
2011[20]\end{array}$ & $\begin{array}{c}\text { women } \\
\text { OSTRA cohort } \\
\text { mean age: } 61 \text { years } \\
\text { age: } 20 \text { to } 70 \text { years }\end{array}$ & $\begin{array}{c}\text { Prospective } \\
102 \text { RA }\end{array}$ & $\begin{array}{c}33 \% \\
\text { IR }=3.7 / 100 \\
\text { person-year } \\
95 \% \text { CI } 2.2 \text { to } 5.8\end{array}$ & & $\begin{array}{c}35 \% \\
\text { IR }=3.7 / 100 \\
\text { person-years } \\
95 \% \text { CI } 2.2 \text { to } 5.8\end{array}$ & \\
\hline $\begin{array}{l}\text { El Maghraoui } \\
\text { Morocco } \\
2015 \text { [16] }\end{array}$ & $\begin{array}{c}\text { men and women }(82.8 \%) \\
\text { mean age: } 54.1 \pm 11.5 \text { years }\end{array}$ & $\begin{array}{c}\text { observational } \\
178 \text { RA }\end{array}$ & $37 \%$ (VFA) & & & \\
\hline $\begin{array}{c}\text { Coulson KA } \\
\text { USA } \\
2009[21]\end{array}$ & $\begin{array}{c}\text { women } \\
\text { CORRONA registry }\end{array}$ & $\begin{array}{l}\text { prospective } \\
8419 \text { RA }\end{array}$ & $\begin{array}{l}\mathrm{IR}=0.78 / 100 \\
\text { person-years }\end{array}$ & $\begin{array}{l}\mathrm{IR}=0.66 / 100 \\
\text { person-years }\end{array}$ & $\begin{array}{c}\mathrm{IR}=2.8 / 100 \\
\text { person-years }\end{array}$ & $\begin{array}{l}\mathrm{IR}=3.71 / 100 \\
\text { person-years }\end{array}$ \\
\hline $\begin{array}{l}\text { Brébant S } \\
\text { France } \\
2012[17]\end{array}$ & $\begin{array}{c}\text { women } \\
56.0 \pm 13.5 \text { years }\end{array}$ & $\begin{array}{c}\text { observational } \\
185 \mathrm{RA}\end{array}$ & $17.8 \%$ (VFA) & & & $31.3 \%$ \\
\hline
\end{tabular}


Table 3. Cont.

\begin{tabular}{|c|c|c|c|c|c|c|}
\hline $\begin{array}{c}\text { Dirven L } \\
\text { The Netherlands } \\
2012 \text { [22] }\end{array}$ & $\begin{array}{c}\text { BeSt study } \\
\text { men and women }(67 \%) \\
\text { mean age: } 54 \text { years }\end{array}$ & $\begin{array}{l}\text { randomized } \\
\text { trial } \\
275 \text { RA }\end{array}$ & $15.0 \%$ & & & \\
\hline $\begin{array}{l}\text { El Maghraoui } \\
\text { Morocco } \\
2015[18]\end{array}$ & $\begin{array}{l}\text { men and women }(82.6 \%) \\
\text { mean age: } 54.1 \pm 11.5 \text { years } \\
\text { age: } 25 \text { to } 82 \text { years }\end{array}$ & $\begin{array}{l}\text { observational } \\
178 \text { RA }\end{array}$ & & & & \\
\hline $\begin{array}{l}\text { Rentero ML } \\
\text { Spain } \\
2015[23]\end{array}$ & $\begin{array}{c}\text { Women } \\
\text { age } \geq 18 \text { years } \\
\text { mean age: } 59.6 \pm 15.0 \text { years }\end{array}$ & $\begin{array}{l}\text { observational } \\
\text { 480 RA }\end{array}$ & $20.0 \%$ (VFA) & & $9.8 \%$ & \\
\hline $\begin{array}{l}\text { Choi YJ } \\
\text { South Korea } \\
2017[24]\end{array}$ & $\begin{array}{c}\text { women } \\
\text { age } \geq 50 \text { years }\end{array}$ & $\begin{array}{c}\text { observational } \\
279 \text { RA }\end{array}$ & $12.5 \%$ & & & \\
\hline $\begin{array}{l}\text { Choi ST } \\
\text { South Korea } \\
2018[19]\end{array}$ & $\begin{array}{c}\text { men and women }(88.9 \%) \\
\text { age: } 61.8 \pm 11.5 \text { years }\end{array}$ & $\begin{array}{l}\text { retrospective } \\
\text { cross-sectional } \\
\text { study } \\
479 \text { RA }\end{array}$ & $16.9 \%$ & $0 \%$ & $0 \%$ & \\
\hline $\begin{array}{l}\text { Phuan-udon R } \\
\text { Thailand } \\
2018 \text { [25] }\end{array}$ & $\begin{array}{c}\text { men and women }(89 \%) \\
\text { Siriraj RA Cohort } \\
\text { mean age: } 61.6 \pm 9.91 \\
\text { age: } 40 \text { to } 90 \text { years }\end{array}$ & 232 RA & & & & $45.7 \%$ \\
\hline
\end{tabular}

Level of significance between RA and non-RA controls: ${ }^{* *} p<0.02 ;{ }^{* * *} p<0.001$; NS: Non-significant; OR: Odd ratio; RR: Relative risk; HR: Hazard ratio; IR: Incidence rate; VFA: Vertebral fracture assessment. 
Table 4. Incidence, prevalence, and risk of fractures in RA patients: Case-control studies.

\begin{tabular}{|c|c|c|c|c|c|c|}
\hline \multirow{2}{*}{$\begin{array}{l}\text { Authors } \\
\text { Country } \\
\text { Year }\end{array}$} & \multirow{2}{*}{ Studied Population } & \multirow{2}{*}{$\begin{array}{c}\text { Type of Study } \\
n \text { RA/ } \\
n \text { Controls }\end{array}$} & \multicolumn{4}{|c|}{ Fractures Sites } \\
\hline & & & Vertebral & Proximal Femur & Non-Vertebral & Any Site \\
\hline $\begin{array}{l}\text { Hooyman JR } \\
\text { USA } \\
1984[26]\end{array}$ & $\begin{array}{l}\text { women } \\
\text { Rochester }\end{array}$ & $\begin{array}{l}\text { population-based } \\
\text { 388 RA }\end{array}$ & $10.8 \%$ & $\begin{array}{c}7.5 \% \\
\text { RR }=1.51 \% * \\
95 \% \text { CI } 1.01 \text { to } 2.17\end{array}$ & $19.6 \%$ & $30.4 \%$ \\
\hline $\begin{array}{l}\text { Verstraeten A } \\
\text { Belgium } \\
1986[27]\end{array}$ & postmenopausal women & $\begin{array}{l}\text { case-control } \\
104 \text { RA } \\
43 \text { controls }\end{array}$ & $7.7 \%$ & $1.0 \%$ & & \\
\hline $\begin{array}{l}\text { Spector } \\
\text { UK } \\
1993[28]\end{array}$ & $\begin{array}{c}\text { postmenopausal women } \\
\text { age } 45 \text { to } 65 \text { years } \\
\text { RA: Five London hospitals } \\
\text { controls: London practice register }\end{array}$ & $\begin{array}{l}\text { case-control } \\
149 \text { RA } \\
713 \text { non-RA controls }\end{array}$ & $\begin{array}{c}12.1 \% \\
\text { OR }=2.1 \\
95 \% \text { CI } 1.2 \text { to } 3.7\end{array}$ & & & \\
\hline $\begin{array}{l}\text { Cooper C } \\
\text { UK } \\
1995[29]\end{array}$ & $\begin{array}{l}\text { men and women }(80 \%) \\
\text { age: } 50 \text { to } 99 \text { years }\end{array}$ & $\begin{array}{l}\text { case-control } \\
300 \text { RA } \\
600 \text { non-RA controls }\end{array}$ & & $\begin{array}{c}\text { OR }=2.195 \% \\
\text { CI } 1.0 \text { to } 4.7 \\
p=0.06 \\
\text { corticosteroids users: } \\
\text { OR }=2.595 \% \\
\text { CI } 1.1 \text { to } 5.5^{*}\end{array}$ & & \\
\hline $\begin{array}{l}\text { Peel NFA } \\
\text { UK } \\
1995[30]\end{array}$ & $\begin{array}{l}\text { postmenopausal women } \\
\text { mean age: } 65 \text { years ( } 50 \text { to } 79 \text { ) years }\end{array}$ & $\begin{array}{l}\text { case-control } \\
\quad 76 \text { RA } \\
\text { corticosteroids users } \\
347 \text { non-RA controls } \\
\end{array}$ & $\begin{array}{l}\quad 27.6 \% \\
\text { OR }=6.295 \% \\
\text { CI } 3.2 \text { to } 12.3\end{array}$ & & & \\
\hline $\begin{array}{c}\text { Martin JC } \\
\text { UK } \\
1997 \\
{[5]}\end{array}$ & $\begin{array}{l}\text { postmenopausal women } \\
\text { from EVOS }\end{array}$ & $\begin{array}{c}\text { case-control } \\
46 \text { RA } \\
29 \text { non-RA controls }\end{array}$ & $\begin{array}{l}\text { corticosteroids } \\
\text { users: } 20 \% \\
\text { non-corticosteroids } \\
\text { users: } 23.8 \%\end{array}$ & & & \\
\hline $\begin{array}{l}\text { Huusko TM } \\
\text { Finland } \\
2001[31]\end{array}$ & $\begin{array}{c}\text { men and women }(72 \%) \\
\text { mean age: } \\
\text { Men } 79 \text { (62 to } 92) \\
\text { women } 77 \text { (52 to } 91)\end{array}$ & $\begin{array}{c}\text { case-control } \\
\text { fractures } \\
\text { Non-RA controls with hip } \\
\text { fractures }\end{array}$ & & $\begin{array}{l}\mathrm{OR}=3.2695 \% \\
\mathrm{CI} 2.26 \text { to } 4.70\end{array}$ & & \\
\hline $\begin{array}{l}\text { Ørstavik RE } \\
\text { Norway } \\
2004[6]\end{array}$ & $\begin{array}{c}\text { women } \\
\text { Oslo RA register } \\
\text { mean age: } 63.0 \text { (50.7 to } 73.6) \text { years }\end{array}$ & $\begin{array}{c}\text { case-control } \\
249 \text { RA } \\
249 \text { non-RA controls }\end{array}$ & $59.0 \%$ & & & \\
\hline
\end{tabular}


Table 4. Cont

\begin{tabular}{|c|c|c|c|c|c|c|}
\hline $\begin{array}{l}\text { Van Staa TP } \\
\text { UK } \\
2006[32]\end{array}$ & $\begin{array}{c}\text { General Practice Research } \\
\text { Database } \\
\text { men and women }(71.1 \%) \\
\geq 40 \text { years }\end{array}$ & $\begin{array}{l}\text { case-control } \\
\text { 30,262 RA } \\
90,783 \text { controls }\end{array}$ & $\begin{array}{c}\mathrm{RR}=2.4 \\
95 \% \text { CI } 2.0 \text { to } 2.8\end{array}$ & $\begin{array}{c}\mathrm{RR}=2.0 \\
95 \% \text { CI } 1.8 \text { to } 2.3\end{array}$ & & $\begin{array}{c}\mathrm{RR}=1.5 \\
95 \% \text { CI } 1.4 \text { to } 1.6\end{array}$ \\
\hline $\begin{array}{l}\text { Kim SY } \\
\text { USA } \\
2010 \\
{[33]}\end{array}$ & $\begin{array}{c}\text { men and women }(73 \%) \\
\text { Health Core Integrated Research } \\
\text { Database } \\
\text { age } \geq 18 \text { years } \\
\text { median age: } 55 \text { years }\end{array}$ & $\begin{array}{c}\text { case-control } \\
\text { 92,827 RA } \\
\text { 921,715 non-RA controls }\end{array}$ & & $\begin{array}{c}\text { men IR }=2.4 / 1000 \\
\text { person-years } \\
\text { women IR }=3.8 / 1000 \\
\text { person-years } \\
\text { HR }=1.44,95 \% \\
\text { CI } 1.24 \text { to } 1.67 \\
\end{array}$ & & $\begin{array}{c}1.4 \% \\
\mathrm{IR}=9.6 / 1000 \text { person-years } \\
\mathrm{HR}=1.2695 \% \text { CI } 1.15 \text { to } 1.38 \\
\text { corticosteroids users: } \\
\mathrm{HR}=1.1595 \% \text { CI } 1.03 \text { to } 1.27\end{array}$ \\
\hline $\begin{array}{l}\text { Wright NC } \\
\text { USA } \\
2011[34]\end{array}$ & $\begin{array}{c}\text { postmenopausal women } \\
50 \text { to } 79 \text { years } \\
\text { WHI study }\end{array}$ & $\begin{array}{c}\text { prospective } \\
\text { 960 RA } \\
83,295 \text { non-RA controls }\end{array}$ & $\begin{array}{c}4.0 \%{ }^{* * *} \\
\text { HR }=1.93^{* * *} \\
95 \% \text { CI } 1.29 \\
\text { to } 2.90\end{array}$ & $\begin{array}{c}4.0 \% * * * \\
\text { IR }=0.51 / 100 \\
\text { person-years } \\
\text { HR }=3.032 .03 \\
\text { to } 4.51 * * *\end{array}$ & & $\begin{array}{c}24.8 \% * * * \\
\mathrm{IR}=3.64 / 100 \text { patient-years } \\
95 \% \text { CI } 3.17 \text { to } 4.11 \\
\text { HR }=1.4995 \% \text { CI } 1.26 \text { to } 1.75 * * *\end{array}$ \\
\hline $\begin{array}{l}\text { Ghazi M } \\
\text { France } \\
2012[35]\end{array}$ & $\begin{array}{c}\text { women } \\
\text { age } 56.1 \pm 14.2 \text { years }\end{array}$ & $\begin{array}{c}\text { case-control } \\
101 \text { RA } \\
303 \text { non-RA controls }\end{array}$ & $\begin{array}{c}21.8 \%(\mathrm{VFA}) * * * \\
\mathrm{OR}=6.595 \% \mathrm{CI} \\
3.1 \text { to } 13.9\end{array}$ & & $28.71 \%$ & \\
\hline $\begin{array}{c}\text { Avouac J } \\
\text { France } \\
2012[9] \\
\end{array}$ & $\begin{array}{c}\text { Women } \\
\text { mean age: } 61 \pm 11 \text { years }\end{array}$ & $\begin{array}{c}\text { case-control } \\
\text { 139 RA } \\
227 \text { non-RA controls } \\
\end{array}$ & $19.0 \% * * *$ & & $22.0 \%$ ** & $33.0 \% * * *$ \\
\hline $\begin{array}{l}\text { Filho JCA } \\
\text { Brazil } \\
2013 \text { [36] }\end{array}$ & $\begin{array}{c}\text { men } \\
\text { age } 51.6 \pm 9.3 \text { years }\end{array}$ & $\begin{array}{c}\text { case-control } \\
50 \text { RA } \\
52 \text { non-RA controls }\end{array}$ & $36.0 \% *$ & & $4 \%$ & \\
\hline $\begin{array}{l}\text { Roussy JP } \\
\text { Canada } \\
2013[37]\end{array}$ & $\begin{array}{c}\text { men and women } \\
\text { Quebec healthcare databases } \\
\text { age } \geq 50 \text { years }\end{array}$ & $\begin{array}{c}\text { case-control } \\
\text { 27,076 RA } \\
\text { non-RA controls }\end{array}$ & & $2.4 \%$ & $\begin{array}{c}5.6 \% \\
\mathrm{IR}=11.0 / 1000 \\
\text { person-years } \\
95 \% \text { CI } 10.4 \\
\text { to } 11.5\end{array}$ & \\
\hline $\begin{array}{l}\text { Brennan SL } \\
\text { Australia } \\
2014[38]\end{array}$ & $\begin{array}{c}\text { women } \\
\text { age } \geq 35 \text { years } \\
\text { database: Barwon Statistical } \\
\text { Division }\end{array}$ & $\begin{array}{c}\text { case-control } \\
1008 \text { RA } \\
172,422 \text { non-RA controls }\end{array}$ & $31.7 \% * * *$ & $7.3 \% * * *$ & & $\begin{array}{c}1.9 \% \\
\mathrm{IR}=114 / 10000 \text { person-years } \\
\mathrm{RR}=1.4395 \% \text { CI } 0.98-2.09 \\
p=0.08\end{array}$ \\
\hline
\end{tabular}


Table 4. Cont.

\begin{tabular}{|c|c|c|c|c|c|}
\hline $\begin{array}{c}\text { Xue A-L } \\
\text { USA, UK, } \\
\text { Sweden, } \\
\text { Norway, } \\
\text { Finland, } \\
\text { Australia, China } \\
2017 \text { [39] }\end{array}$ & men and women & meta-analysis (13 studies) & $\begin{array}{l}\mathrm{RR}=2.9395 \% \\
\mathrm{CI} 2.25 \text { to } 3.83\end{array}$ & $\begin{array}{c}\mathrm{RR}=2.4195 \% \text { CI } 1.83 \\
\text { to } 3.17\end{array}$ & $\mathrm{RR}=2.2595 \%$ CI 1.76 to 2.87 \\
\hline $\begin{array}{l}\text { Clynes MA } \\
\text { UK } \\
2019[40]\end{array}$ & $\begin{array}{c}\text { men and women }(70.1 \%) \\
\text { UK Biobank: Hospital Episode } \\
\text { Statistics (HES) }\end{array}$ & $\begin{array}{c}\text { case-control } \\
5492 \text { RA } \\
497,051 \text { non-RA controls }\end{array}$ & & & $\begin{array}{c}\text { men: } 5.1 \% \\
\text { OR }=1.4695 \% \text { CI } 1.17 \text { to } 1.81 * * * \\
\text { women: } 5.5 \% \\
\text { OR }=1.7195 \% \text { CI } 1.50 \text { to } 1.94 * * *\end{array}$ \\
\hline $\begin{array}{c}\text { Hong } \\
\text { Taiwan } \\
2019[41]\end{array}$ & $\begin{array}{l}\text { men and women } \\
\text { Nationwide database } \\
\text { age } \geq 40 \text { years }\end{array}$ & $\begin{array}{l}\text { retrospective } \\
30.507 \mathrm{RA}\end{array}$ & $\begin{array}{l}\mathrm{HR}=1.47^{* * *} \\
95 \% \mathrm{CI} 1.19 \\
\text { to } 1.81\end{array}$ & & \\
\hline $\begin{array}{l}\text { Weiss RJ } \\
\text { Sweden } \\
2019[42]\end{array}$ & $\begin{array}{c}\text { men and women }(66 \%) \\
\text { Swedish National Hospital } \\
\text { Discharge Register } \\
\text { median age: } 71 \text { years }\end{array}$ & $\begin{array}{c}\text { case-control } \\
\text { 3379 RA } \\
\text { 420,331 non-RA controls }\end{array}$ & $\begin{array}{c}\mathrm{OI}=2.7 * 95 \% \\
\mathrm{CI} 2.1 \text { to } 3.4\end{array}$ & $\begin{array}{c}\mathrm{OI}=2.9 * 95 \% \mathrm{CI} 2.7 \\
\text { to } 3.1\end{array}$ & $\mathrm{OI}=2.9 * 95 \% \mathrm{CI} 2.8$ to 3.1 \\
\hline
\end{tabular}


Cohort and registry studies allow calculating the incidence-rates of fractures mostly for peripheral fractures. A Canadian nested case-control study conducted using Quebec physician billing, pharmacy data from the "Régie de l'assurance maladie du Québec", and hospital discharge data found that the incidence rate of non-vertebral osteoporotic fractures is 11.0/1000 person-years in the population of RA patients aged 50 years and more [37]. In another large study conducted based on a USA health database on both men and women aged more than 18 years comparing 92,827 RA to 921,715 non-RA controls, the incidence rate of fracture at any of the four sites (wrist, humerus, hip, and pelvis) among RA patients was 9.6 per 1000 person-years and 1.5 times higher than that of non-RA patients (6.3 per 1000 person-years) [33].

In contrast with other peripheral sites, the incidence of wrist fracture decreased in RA as compared to the controls [6,32]. It could be due to different risk factors, especially for the mechanisms of fall. It was expected to find the same risk factors for a fracture in the RA population and the general population. Patients with incident vertebral deformities or peripheral fractures are significantly older [16,22], had lower BMD, higher disability and more often a previous fracture and a higher cumulative steroid [11,15,20], frequent falls [35], low body mass index (BMI), alcohol abuse, smoking [28], risk factors resulting from the disease itself such as the intensity of the inflammation and disability $[29,36,40]$, duration of the disease [1,37], and cachexia [18].

Similar to the general population, patients with a morphometric, self-reported vertebral fracture or self-reported non-vertebral fracture have a higher statistically significant 10-year probability of sustaining a major osteoporotic fracture or hip fracture than patients without fractures [23].

According to Avouac et al. [9], BMD incompletely predicts patients who will suffer from an osteoporotic fracture, since only half of RA patients with fractures had BMD $\leq-2.5 \mathrm{SD}$. A higher proportion of RA patients have vertebral fractures, although their BMD is above the osteoporotic threshold. These data suggest that other parameters, especially the bone microarchitecture, have to be considered and underlines the need for other techniques and strategies to assess the risk of fracture in RA, such as the FRAXtool ${ }^{\circledR}$ or the trabecular bone score (TBS). The fracture risk assessment tool ${ }^{\circledR}$ developed by the World Health Organization (FRAXtool ${ }^{\circledR}$ ) [48] computes the 10-year probability of a hip fracture or a major osteoporotic fracture. It integrates the risks associated with BMD at the femoral neck as well as clinical risk factors such as age, height, weight, sex, smoking status, alcohol drinking, use of glucocorticoids, and history of secondary osteoporosis. FRAXtool ${ }^{\circledR}$ is useful to set a threshold in order to screen candidates for pharmacological intervention and the proportion of RA patients with a greater risk of osteoporotic fractures detected with FRAXtool ${ }^{\circledR}$ is higher than with the WHO criteria [19]. When applied to 238 patients enrolled in the Canadian Early Arthritis Cohort (CATCH) study without using BMD measurements, FRAXtool ${ }^{\circledR}$ identified $5 \%$ to $13 \%$ of patients at a high risk for fracture [49]. Patients with a higher osteoporotic fracture risk determined by FRAXtool ${ }^{\circledR}$ are more likely to be female, especially postmenopausal women, with alcohol use, glucocorticoid use, proton pump inhibitor use, and had lower BMI $\left(<25 \mathrm{~kg} / \mathrm{m}^{2}\right)$ [19]. High fracture risk patients identified by the FRAXtool ${ }^{\circledR}$ was also statistically associated with disease duration, menopause duration, disease activity score (DAS28), and health assessment questionnaire (HAQ) [25].

The trabecular bone score (TBS) is also a useful, new tool to improve the prediction of fragility fracture. It is a parameter that can be automatically calculated during a BMD measurement. It assesses the quality of bone texture using the pixel gray-level variations in dual-energy X-ray absorptiometry images. A low TBS value suggests a low quality of bone texture. In the RA population, a low TBS is associated with a higher risk of having vertebral fractures even after adjusting for confounding factors such as age, DAS28, and BMD $[17,24]$. TBS can now be combined with the FRAX tool ${ }^{\circledR}$. The TBS could be a supplementary tool for discriminating osteoporotic fractures risk in postmenopausal women with RA. It may have a nonlinear relationship with the cumulative dose of GCs, but not with the RA disease activity. 


\section{Physiopathology of Bone Loss in Rheumatoid Arthritis}

As expected, classical risk factors for osteoporosis are still valuable to predict osteoporosis in patients with RA. In most of the studies, patients with spinal or femoral osteoporosis were more often female than male [3], were older [26], have a previous history of low trauma fracture, [12,17] had a lower BMI, a longer disease duration [10], a corticosteroid treatment [11], a high Larsen score [14], and a higher HAQ score $[11,23,29]$. There is an excess of bone loss among RA women compared to controls with post-menopausal status and HRT reduces bone resorption regardless of glucocorticoid therapy in post-menopausal RA patients [2]. There could also be an interaction between estrogen deficiency and RA-genetic risk alleles promoting enhanced Th17-cell autoreactivity, manifested by ACPA (+) RA that exacerbates the inflammatory conditions and enhances loss.

In addition to these classical risk factors, there is a crosstalk between bone cells and the immunity system and chronic inflammation that can perturb bone metabolism and promote an increase in bone loss. There is a consistent association between bone loss and the CRP reflecting disease activity $[3,20,50]$.

The association between joint damage progression and both hand and generalized BMD loss in RA suggests common mechanisms, with hand BMD loss occurring earlier than generalized BMD loss in the disease course [51,52].

In the CATCH study, the disease activity score with 28 joint counts in the high-risk fracture group was significantly higher compared to the low-risk fracture group $(p=0.048)$ [49].

\subsection{Role of Cytokines}

Pro-inflammatory cytokines, particularly tumor necrosis factor (TNF) and interleukins IL-1, IL-6, and IL-17 play a key role in the pathogenesis of inflammation-induced bone loss. The TNF- $\alpha$ stimulates the receptor activator of nuclear factor kappa B ligand (RANKL), which is a membrane protein secreted by osteoblasts that binds to the RANK receptor on osteoclast precursors and induces osteoclast maturation and activation leading to bone resorption and inhibits osteoblast function thus bone formation [53]. In addition, osteoprotegerin (OPG) acts as a decoy receptor, the RANKL/OPG being of major importance to assess the balance between bone formation and resorption. OPG expression is increased in anti-TNF treated patients while RANKL is not decreased even if its level can predict the therapeutic response to anti-TNF therapy.

In mice, despite TNF-mediated inflammatory arthritis, systemic bone is fully protected by the absence of interleukin-1 (IL-1), which suggests that IL-1 is an essential mediator of inflammatory osteopenia [54]. Thus, both TNF- $\alpha$ and IL1 play a key role in the pathogenesis of inflammation-induced bone loss in RA patients.

The Wnt/B catenin pathway is a crucial regulatory pathway for bone formation inhibiting osteoblastic differentiation and activity. Dickkopf-1 (Dkk-1) and sclerostin (SCL) are blockers of the Wnt-signaling pathway and play an important role in RA. TNF- $\alpha$ increases Dkk-1 which down regulates the Wnt pathway, blocking osteoblast differentiation and inducing expression of SCL leading to the apoptosis of osteocytes.

\subsection{Bone Remodeling Markers in Ra}

Histomorphometric analysis from trans-iliac bone biopsies show that the low bone mass was not only due to an increased bone resorption but also due to reduced bone formation [55]. These mechanisms of bone loss have been assessed in many studies [56] by measuring bone remodeling markers: Osteocalcin, bone alkaline phosphatase (BAP), carboxy-terminal collagen cross links (CTX), carboxy-terminal propeptides of type 1 collagen (ICTP), and deoxypyridinoline. These bone remodeling markers are surrogates to evaluate bone formation, resorption, and further risk of fractures. I-CTX, which is derived from a matrix metalloprotease activity (MMPs), reflects joint erosions while I-CTX, produced by cathepsin K, gives evidence of general osteoporosis. 
In the COBRA study, high levels of measured I-CTX and II-CTX were strong and independent predictors of articular damages [57].

\section{Effects on Bone of Treatments for Rheumatoid Arthritis}

The DMARDs reduce the inflammation and by the way are associated with a reduced bone loss in RA patients [40]. In some studies, BMD is decreased in RA according to corticosteroid usage, especially at the lumbar spine in both females and males with RA, and in those who are current users of steroids $[1,9,11]$ with an increased risk of fracture [21]. The effect of corticosteroid may vary according to skeletal sites [4].

\subsection{Corticosteroids}

Other authors were not able to show a detrimental effect of corticosteroids on bone in RA patients $[32,58]$ and some showed even a protective effect probably mediated by a better control of inflammation [35], which is known to be associated with a decrease in structural damage and bone loss $[59,60]$.

In the Canadian Early Arthritis Cohort (CATCH), there was a significant correlation between increased fracture risk groups measured by the FRAXtool ${ }^{\circledR}$ and oral glucocorticoid use $(p=0.012)$ and baseline erosions $(p=0.040)$ [49].

The use of corticosteroids may be an indicator of more severe disease and a poorer functional status if the degree of systemic inflammation and the severity of RA correlated with the risk of fracture. Their detrimental effect on the fracture might have been confounded if the drug was selectively given to patients with a higher degree of systemic inflammation and RA severity [33].

In contrast, several studies recently reported a potentially beneficial effect of low-dose, short-term systemic glucocorticoids on BMD in RA suggesting that the deleterious effect of prednisolone on bone may be counteracted by its anti-inflammatory effect $[47,60,61]$.

Other drugs, used specifically or not for RA, have been suspected to be detrimental for bone such as opioids, selective serotonin reuptake inhibitors (SSRIs), and anticonvulsants. All were associated with an increased risk of fracture in patients with RA [37,62].

\subsection{Biological Agents}

In RA patients, BMD is inversely correlated to serum levels of TNF- $\alpha$ and an optimal control of inflammation has been shown to reduce bone loss in this inflammatory disease.

Some observational or randomized study designs have reported that inflammatory generalized bone loss is suppressed by biological DMARDs targeting pro-inflammatory mediators such as the TNF- $\alpha$ (adalimumab, certolizumab, etanercept, golimumab, and infliximab), CD80/CD86 (abatacept), IL-6 (tocilizumab), CD20 (rituximab), and IL-1 (anakinra) both at the hip and lumbar spine. Nevertheless, this global protective effect was associated with a continuing bone loss in the BMD of the hands, which could reflect a suboptimal suppression of local inflammation [63-65]. This effect on BMD is accompanied by a significant increase in osteocalcin serum levels and a significant decrease in markers for bone resorption [66].

\section{Conclusions}

Bone loss in RA patients is well documented and is a frequent comorbidity. Fractures resulting from osteoporosis ranked high among comorbidities in contributing to mortality, future hospitalizations, and increased disability. Regular bone mineral density (BMD) measurements and fracture risk assessments using tools such as the FRAX algorithm should be performed for an early detection of osteoporosis in RA patients. Furthermore, the VFA technology on DXA devices should be used in these patients at the time of BMD measurement. We presume that with a lesser use of corticosteroids due to a better control of the inflammation and the potential protective effect of biologic agents that the prevalence of osteoporosis will slightly decrease. However, the risk 
of fractures remains high in this population highlighting the need for monitoring osteoporosis among RA patients and justifying its prevention and treatment.

Author Contributions: Writing—original draft, writing—review and editing, P.F.; co-writing—original draft, supervision, writing — review and editing, E.S.; writing—review and editing, L.L.M.; supervision, writing-review and editing, V.G. All authors have read and agreed to the published version of the manuscript.

Funding: This research received no external funding.

Conflicts of Interest: The authors declare no conflict of interest.

\section{References}

1. Garton:, M.J.; Reid, D.M. Bone Mineral Density of the Hip and of the Anteroposterior and Lateral Dimensions of the Spine in Men with Rheumatoid Arthritis: Effects of Low-Dose Corticosteroids. Arthritis Rheum. 1993, 36, 222-228. [CrossRef] [PubMed]

2. Hall, G.M.; Spector, T.D.; Jane Griffin, A.; SM Jawad, A.; Hall, M.L.; Doyle, D.V. The Effect of Rheumatoid Arthritis and Steroid Therapy on Bone Density in Postmenopausal Women. Arthritis Rheum. 1993, 36, 1510-1516. [CrossRef] [PubMed]

3. Gough, A. Generalised Bone Loss in Patients with Early Rheumatoid Arthritis. The Lancet 1994, 344, $23-27$. [CrossRef]

4. Lane, N.E.; Pressman, A.R.; Star, V.L.; Cummings, S.R.; Nevitt, M.C. Rheumatoid Arthritis and Bone Mineral Density in Elderly Women. The Study of Osteoporotic Fractures Research Group. J. Bone Miner. Res. Off. J. Am. Soc. Bone Miner. Res. 1995, 10, 257-263. [CrossRef]

5. Martin, J.C.; Munro, R.; Campbell, M.K.; Reid, D.M. Effects of Disease and Corticosteroids on Appendicular Bone Mass in Postmenopausal Women with Rheumatoid Arthritis: Comparison with Axial Measurements. Br. J. Rheumatol. 1997, 36, 43-49. [CrossRef]

6. Ørstavik, R.E.; Haugeberg, G.; Mowinckel, P.; Høiseth, A.; Uhlig, T.; Falch, J.A.; Halse, J.I.; McCloskey, E.; Kvien, T.K. Vertebral Deformities in Rheumatoid Arthritis: A Comparison with Population-Based Controls. Arch. Intern. Med. 2004, 164, 420-425. [CrossRef]

7. Haugeberg, G.; Uhlig, T.; Falch, J.A.; Halse, J.I.; Kvien, T.K. Reduced Bone Mineral Density in Male Rheumatoid Arthritis Patients: Frequencies and Associations with Demographic and Disease Variables in Ninety-Four Patients in the Oslo County Rheumatoid Arthritis Register. Arthritis Rheum. 2000, 43, 2776-2784. [CrossRef]

8. Lee, S.-G.; Park, Y.-E.; Park, S.-H.; Kim, T.-K.; Choi, H.-J; Lee, S.-J.; Kim, S.-I.; Lee, S.-H.; Kim, G.-T.; Lee, J.-W.; et al. Increased Frequency of Osteoporosis and BMD below the Expected Range for Age among South Korean Women with Rheumatoid Arthritis. Int. J. Rheum. Dis. 2012, 15, 289-296. [CrossRef]

9. Avouac, J.; Koumakis, E.; Toth, E.; Meunier, M.; Maury, E.; Kahan, A.; Cormier, C.; Allanore, Y. Increased Risk of Osteoporosis and Fracture in Women with Systemic Sclerosis: A Comparative Study with Rheumatoid Arthritis. Arthritis Care Res. 2012, 64, 1871-1878. [CrossRef]

10. Laan, R.F.; Buijs, W.C.; Verbeek, A.L.; Draad, M.P.; Corstens, F.H.; van de Putte, L.B.; van Riel, P.L. Bone Mineral Density in Patients with Recent Onset Rheumatoid Arthritis: Influence of Disease Activity and Functional Capacity. Ann. Rheum. Dis. 1993, 52, 21-26. [CrossRef]

11. Sinigaglia, L.; Nervetti, A.; Mela, Q.; Bianchi, G.; Del Puente, A.; Di Munno, O.; Frediani, B.; Cantatore, F.; Pellerito, R.; Bartolone, S.; et al. A Multicenter Cross Sectional Study on Bone Mineral Density in Rheumatoid Arthritis. Italian Study Group on Bone Mass in Rheumatoid Arthritis. J. Rheumatol. 2000, 27, 2582-2589. [PubMed]

12. Kvien, T.K.; Haugeberg, G.; Uhlig, T.; Falch, J.A.; Halse, J.I.; Lems, W.F.; Dijkmans, B.A.; Woolf, A.D. Data Driven Attempt to Create a Clinical Algorithm for Identification of Women with Rheumatoid Arthritis at High Risk of Osteoporosis. Ann. Rheum. Dis. 2000, 59, 805-811. [CrossRef] [PubMed]

13. Haugeberg, G.; Uhlig, T.; Falch, J.A.; Halse, J.I.; Kvien, T.K. Bone Mineral Density and Frequency of Osteoporosis in Female Patients with Rheumatoid Arthritis: Results from 394 Patients in the Oslo County Rheumatoid Arthritis Register. Arthritis Rheum. 2000, 43, 522-530. [CrossRef] 
14. Lodder, M.C.; Haugeberg, G.; Lems, W.F.; Uhlig, T.; Orstavik, R.E.; Kostense, P.J.; Dijkmans, B.A.C.; Kvien, T.K.; Woolf, A.D.; Oslo-Truro-Amsterdam (OSTRA) Collaborative Study. Radiographic Damage Associated with Low Bone Mineral Density and Vertebral Deformities in Rheumatoid Arthritis: The Oslo-Truro-Amsterdam (OSTRA) Collaborative Study. Arthritis Rheum. 2003, 49, 209-215. [CrossRef] [PubMed]

15. Ørstavik, R.E.; Haugeberg, G.; Uhlig, T.; Falch, J.A.; Halse, J.I.; Høiseth, A.; Lilleås, F.; Kvien, T.K. Vertebral Deformities in 229 Female Patients with Rheumatoid Arthritis: Associations with Clinical Variables and Bone Mineral Density. Arthritis Rheum. 2003, 49, 355-360. [CrossRef] [PubMed]

16. El Maghraoui, A.; Rezqi, A.; Mounach, A.; Achemlal, L.; Bezza, A.; Ghozlani, I. Prevalence and Risk Factors of Vertebral Fractures in Women with Rheumatoid Arthritis Using Vertebral Fracture Assessment. Rheumatol. Oxf. Engl. 2010, 49, 1303-1310. [CrossRef]

17. Bréban, S.; Briot, K.; Kolta, S.; Paternotte, S.; Ghazi, M.; Fechtenbaum, J.; Roux, C. Identification of Rheumatoid Arthritis Patients With Vertebral Fractures Using Bone Mineral Density and Trabecular Bone Score. J. Clin. Densitom. 2012, 15, 260-266. [CrossRef]

18. El Maghraoui, A.; Sadni, S.; Rezqi, A.; Bezza, A.; Achemlal, L.; Mounach, A. Does Rheumatoid Cachexia Predispose Patients with Rheumatoid Arthritis to Osteoporosis and Vertebral Fractures? J. Rheumatol. 2015, 42, 1556-1562. [CrossRef]

19. Choi, S.; Kwon, S.-R.; Jung, J.-Y.; Kim, H.-A.; Kim, S.-S.; Kim, S.; Kim, J.-M.; Park, J.-H.; Suh, C.-H. Prevalence and Fracture Risk of Osteoporosis in Patients with Rheumatoid Arthritis: A Multicenter Comparative Study of the FRAX and WHO Criteria. J. Clin. Med. 2018, 7, 507. [CrossRef]

20. Vis, M.; Haavardsholm, E.A.; Bøyesen, P.; Haugeberg, G.; Uhlig, T.; Hoff, M.; Woolf, A.; Dijkmans, B.; Lems, W.; Kvien, T.K. High Incidence of Vertebral and Non-Vertebral Fractures in the OSTRA Cohort Study: A 5-Year Follow-up Study in Postmenopausal Women with Rheumatoid Arthritis. Osteoporos. Int. J. Establ. Result Coop. Eur. Found. Osteoporos. Natl. Osteoporos. Found. USA 2011, 22, 2413-2419. [CrossRef]

21. Coulson, K.A.; Reed, G.; Gilliam, B.E.; Kremer, J.M.; Pepmueller, P.H. Factors Influencing Fracture Risk, T Score, and Management of Osteoporosis in Patients with Rheumatoid Arthritis in the Consortium of Rheumatology Researchers of North America (CORRONA) Registry. J. Clin. Rheumatol. Pract. Rep. Rheum. Musculoskelet. Dis. 2009, 15, 155-160. [CrossRef] [PubMed]

22. Dirven, L.; van den Broek, M.; van Groenendael, J.H.L.M.; de Beus, W.M.; Kerstens, P.J.S.M.; Huizinga, T.W.J.; Allaart, C.F.; Lems, W.F. Prevalence of Vertebral Fractures in a Disease Activity Steered Cohort of Patients with Early Active Rheumatoid Arthritis. BMC Musculoskelet. Disord. 2012, 13, 125. [CrossRef] [PubMed]

23. Rentero, M.L.; Amigo, E.; Chozas, N.; Fernández Prada, M.; Silva-Fernández, L.; Abad Hernandez, M.A.; Rodriguez Barrera, J.M.; del Pino-Montes, J.; GHDP Study Group. Prevalence of Fractures in Women with Rheumatoid Arthritis and/or Systemic Lupus Erythematosus on Chronic Glucocorticoid Therapy. BMC Musculoskelet. Disord. 2015, 16, 300. [CrossRef] [PubMed]

24. Choi, Y.J.; Chung, Y.-S.; Suh, C.-H.; Jung, J.-Y.; Kim, H.-A. Trabecular Bone Score as a Supplementary Tool for the Discrimination of Osteoporotic Fractures in Postmenopausal Women with Rheumatoid Arthritis. Medicine 2017, 96, e8661. [CrossRef]

25. Phuan-Udom, R.; Lektrakul, N.; Katchamart, W. The Association between 10-Year Fracture Risk by FRAX and Osteoporotic Fractures with Disease Activity in Patients with Rheumatoid Arthritis. Clin. Rheumatol. 2018, 37, 2603-2610. [CrossRef]

26. Hooyman, J.R.; Melton, L.J.; Nelson, A.M.; O'Fallon, W.M.; Riggs, B.L. Fractures after Rheumatoid Arthritis. A Population-Based Study. Arthritis Rheum. 1984, 27, 1353-1361. [CrossRef]

27. Verstraeten, A.; Dequeker, J. Vertebral and Peripheral Bone Mineral Content and Fracture Incidence in Postmenopausal Patients with Rheumatoid Arthritis: Effect of Low Dose Corticosteroids. Ann. Rheum. Dis. 1986, 45, 852-857. [CrossRef]

28. Spector, T.D.; Hall, G.M.; McCloskey, E.V.; Kanis, J.A. Risk of Vertebral Fracture in Women with Rheumatoid Arthritis. BMJ 1993, 306, 558. [CrossRef]

29. Cooper, C.; Coupland, C.; Mitchell, M. Rheumatoid Arthritis, Corticosteroid Therapy and Hip Fracture. Ann. Rheum. Dis. 1995, 54, 49-52. [CrossRef] 
30. Peel, N.F.; Moore, D.J.; Barrington, N.A.; Bax, D.E.; Eastell, R. Risk of Vertebral Fracture and Relationship to Bone Mineral Density in Steroid Treated Rheumatoid Arthritis. Ann. Rheum. Dis. 1995, 54, 801-806. [CrossRef]

31. Huusko, T.M.; Korpela, M.; Karppi, P.; Avikainen, V.; Kautiainen, H.; Sulkava, R. Threefold Increased Risk of Hip Fractures with Rheumatoid Arthritis in Central Finland. Ann. Rheum. Dis. 2001, 60, 521-522. [CrossRef] [PubMed]

32. van Staa, T.P.; Geusens, P.; Bijlsma, J.W.J.; Leufkens, H.G.M.; Cooper, C. Clinical Assessment of the Long-Term Risk of Fracture in Patients with Rheumatoid Arthritis. Arthritis Rheum. 2006, 54, 3104-3112. [CrossRef] [PubMed]

33. Kim, S.Y.; Schneeweiss, S.; Liu, J.; Daniel, G.W.; Chang, C.-L.; Garneau, K.; Solomon, D.H. Risk of Osteoporotic Fracture in a Large Population-Based Cohort of Patients with Rheumatoid Arthritis. Arthritis Res. Ther. 2010, 12, R154. [CrossRef] [PubMed]

34. Wright, N.C.; Lisse, J.R.; Walitt, B.T.; Eaton, C.B.; Chen, Z.; Women's Health Initiative Investigators. Arthritis Increases the Risk for Fractures-Results from the Women's Health Initiative. J. Rheumatol. 2011, 38, 1680-1688. [CrossRef]

35. Ghazi, M.; Kolta, S.; Briot, K.; Fechtenbaum, J.; Paternotte, S.; Roux, C. Prevalence of Vertebral Fractures in Patients with Rheumatoid Arthritis: Revisiting the Role of Glucocorticoids. Osteoporos. Int. J. Establ. Result Coop. Eur. Found. Osteoporos. Natl. Osteoporos. Found. USA 2012, 23, 581-587. [CrossRef] [PubMed]

36. Filho, J.C.A.; Pinheiro, M.M.; de Moura Castro, C.H.; Szejnfeld, V.L. Prevalence and Risk Factors Associated with Low-Impact Fractures in Men with Rheumatoid Arthritis. Clin. Rheumatol. 2014, 33, 1389-1395. [CrossRef]

37. Roussy, J.-P.; Bessette, L.; Bernatsky, S.; Rahme, E.; Lachaine, J. Biologic Disease-Modifying Anti-Rheumatic Drugs and the Risk of Non-Vertebral Osteoporotic Fractures in Patients with Rheumatoid Arthritis Aged 50 Years and Over. Osteoporos. Int. J. Establ. Result Coop. Eur. Found. Osteoporos. Natl. Osteoporos. Found. USA 2013, 24, 2483-2492. [CrossRef]

38. Brennan, S.L.; Toomey, L.; Kotowicz, M.A.; Henry, M.J.; Griffiths, H.; Pasco, J.A. Rheumatoid Arthritis and Incident Fracture in Women: A Case-Control Study. BMC Musculoskelet. Disord. 2014, 15, 13. [CrossRef]

39. Xue, A.-L.; Wu, S.-Y.; Jiang, L.; Feng, A.-M.; Guo, H.-F.; Zhao, P. Bone Fracture Risk in Patients with Rheumatoid Arthritis: A Meta-Analysis. Medicine 2017, 96, e6983. [CrossRef]

40. Clynes, M.A.; Jameson, K.; Prieto-Alhambra, D.; Harvey, N.C.; Cooper, C.; Dennison, E.M. Impact of Rheumatoid Arthritis and Its Management on Falls, Fracture and Bone Mineral Density in UK Biobank. Front. Endocrinol. 2019, 10, 817. [CrossRef]

41. Hong, W.-J.; Chen, W.; Yeo, K.-J.; Huang, P.-H.; Chen, D.-Y.; Lan, J.-L. Increased Risk of Osteoporotic Vertebral Fracture in Rheumatoid Arthritis Patients with New-Onset Cardiovascular Diseases: A Retrospective Nationwide Cohort Study in Taiwan. Osteoporos. Int. J. Establ. Result Coop. Eur. Found. Osteoporos. Natl. Osteoporos. Found. USA 2019, 30, 1617-1625. [CrossRef] [PubMed]

42. Weiss, R.J.; Wick, M.C.; Ackermann, P.W.; Montgomery, S.M. Increased Fracture Risk in Patients with Rheumatic Disorders and Other Inflammatory Diseases-A Case-Control Study with 53,108 Patients with Fracture. J. Rheumatol. 2010, 37, 2247-2250. [CrossRef] [PubMed]

43. Bultink, I.E.M.; Vis, M.; van der Horst-Bruinsma, I.E.; Lems, W.F. Inflammatory Rheumatic Disorders and Bone. Curr. Rheumatol. Rep. 2012, 14, 224-230. [CrossRef] [PubMed]

44. Michaud, K.; Wolfe, F. Comorbidities in Rheumatoid Arthritis. Best Pract. Res. Clin. Rheumatol. 2007, 21, 885-906. [CrossRef]

45. Schett, G.; Kiechl, S.; Weger, S.; Pederiva, A.; Mayr, A.; Petrangeli, M.; Oberhollenzer, F.; Lorenzini, R.; Redlich, K.; Axmann, R.; et al. High-Sensitivity C-Reactive Protein and Risk of Nontraumatic Fractures in the Bruneck Study. Arch. Intern. Med. 2006, 166, 2495-2501. [CrossRef]

46. Güler-Yüksel, M.; Allaart, C.F.; Goekoop-Ruiterman, Y.P.M.; de Vries-Bouwstra, J.K.; van Groenendael, J.H.L.M.; Mallée, C.; de Bois, M.H.W.; Breedveld, F.C.; Dijkmans, B.A.C.; Lems, W.F. Changes in Hand and Generalised Bone Mineral Density in Patients with Recent-Onset Rheumatoid Arthritis. Ann. Rheum. Dis. 2009, 68, 330-336. [CrossRef] 
47. Engvall, I.-L.; Svensson, B.; Tengstrand, B.; Brismar, K.; Hafström, I.; Better Anti-Rheumatic FarmacO Therapy Study Group. Impact of Low-Dose Prednisolone on Bone Synthesis and Resorption in Early Rheumatoid Arthritis: Experiences from a Two-Year Randomized Study. Arthritis Res. Ther. 2008, 10, R128. [CrossRef]

48. Kanis, J.A.; McCloskey, E.V.; Johansson, H.; Oden, A.; Ström, O.; Borgström, F. Development and Use of FRAX in Osteoporosis. Osteoporos. Int. J. Establ. Result Coop. Eur. Found. Osteoporos. Natl. Osteoporos. Found. USA 2010, 21 (Suppl. 2), 407-413. [CrossRef]

49. Cheng, C.K.; McDonald-Blumer, H.; Boire, G.; Pope, J.E.; Haraoui, B.; Hitchon, C.A.; Thorne, C.; Sun, Y.; Bykerk, V.P. Care Gap in Patients with Early Inflammatory Arthritis with a High Fracture Risk Identified Using FRAX(®). J. Rheumatol. 2010, 37, 2221-2225. [CrossRef]

50. Cauley, J.A.; Danielson, M.E.; Boudreau, R.M.; Forrest, K.Y.Z.; Zmuda, J.M.; Pahor, M.; Tylavsky, F.A.; Cummings, S.R.; Harris, T.B.; Newman, A.B. Inflammatory Markers and Incident Fracture Risk in Older Men and Women: The Health Aging and Body Composition Study. J. Bone Miner. Res. 2007, 22, 1088-1095. [CrossRef]

51. Takayanagi, H. Osteoimmunology and the Effects of the Immune System on Bone. Nat. Rev. Rheumatol. 2009, 5, 667-676. [CrossRef] [PubMed]

52. Kleyer, A.; Finzel, S.; Rech, J.; Manger, B.; Krieter, M.; Faustini, F.; Araujo, E.; Hueber, A.J.; Harre, U.; Engelke, K.; et al. Bone Loss before the Clinical Onset of Rheumatoid Arthritis in Subjects with Anticitrullinated Protein Antibodies. Ann. Rheum. Dis. 2014, 73, 854-860. [CrossRef] [PubMed]

53. Redlich, K.; Smolen, J.S. Inflammatory Bone Loss: Pathogenesis and Therapeutic Intervention. Nat. Rev. Drug Discov. 2012, 11, 234-250. [CrossRef]

54. Polzer, K.; Joosten, L.; Gasser, J.; Distler, J.H.; Ruiz, G.; Baum, W.; Redlich, K.; Bobacz, K.; Smolen, J.S.; van den Berg, W.; et al. Interleukin-1 Is Essential for Systemic Inflammatory Bone Loss. Ann. Rheum. Dis. 2010, 69, 284-290. [CrossRef] [PubMed]

55. Compston, J.E.; Vedi, S.; Croucher, P.I.; Garrahan, N.J.; O'Sullivan, M.M. Bone Turnover in Non-Steroid Treated Rheumatoid Arthritis. Ann. Rheum. Dis. 1994, 53, 163-166. [CrossRef] [PubMed]

56. Fardellone, P.; Séjourné, A.; Paccou, J.; Goëb, V. Bone Remodelling Markers in Rheumatoid Arthritis. Mediators Inflamm. 2014, 2014, 484280. [CrossRef] [PubMed]

57. van Tuyl, L.H.D.; Voskuyl, A.E.; Boers, M.; Geusens, P.; Landewé, R.B.M.; Dijkmans, B.A.C.; Lems, W.F. Baseline RANKL:OPG Ratio and Markers of Bone and Cartilage Degradation Predict Annual Radiological Progression over 11 Years in Rheumatoid Arthritis. Ann. Rheum. Dis. 2010, 69, 1623-1628. [CrossRef]

58. Kröger, H.; Honkanen, R.; Saarikoski, S.; Alhava, E. Decreased Axial Bone Mineral Density in Perimenopausal Women with Rheumatoid Arthritis-a Population Based Study. Ann. Rheum. Dis. 1994, 53, 18-23. [CrossRef] [PubMed]

59. Roux, C. Osteoporosis in Inflammatory Joint Diseases. Osteoporos. Int. J. Establ. Result Coop. Eur. Found. Osteoporos. Natl. Osteoporos. Found. USA 2011, 22, 421-433. [CrossRef] [PubMed]

60. Korczowska, I.; Olewicz-Gawlik, A.; Trefler, J.; Hrycaj, P.; Krzysztof Łacki, J. Does Low-Dose and Short-Term Glucocorticoids Treatment Increase the Risk of Osteoporosis in Rheumatoid Arthritis Female Patients? Clin. Rheumatol. 2008, 27, 565-572. [CrossRef]

61. Haugeberg, G.; Strand, A.; Kvien, T.K.; Kirwan, J.R. Reduced Loss of Hand Bone Density with Prednisolone in Early Rheumatoid Arthritis: Results from a Randomized Placebo-Controlled Trial. Arch. Intern. Med. 2005, 165, 1293-1297. [CrossRef]

62. Ozen, G.; Pedro, S.; Wolfe, F.; Michaud, K. Medications Associated with Fracture Risk in Patients with Rheumatoid Arthritis. Ann. Rheum. Dis. 2019, 78, 1041-1047. [CrossRef] [PubMed]

63. Vis, M.; Havaardsholm, E.A.; Haugeberg, G.; Uhlig, T.; Voskuyl, A.E.; van de Stadt, R.J.; Dijkmans, B.A.C.; Woolf, A.D.; Kvien, T.K.; Lems, W.F. Evaluation of Bone Mineral Density, Bone Metabolism, Osteoprotegerin and Receptor Activator of the NFkappaB Ligand Serum Levels during Treatment with Infliximab in Patients with Rheumatoid Arthritis. Ann. Rheum. Dis. 2006, 65, 1495-1499. [CrossRef] [PubMed]

64. Wijbrandts, C.A.; Klaasen, R.; Dijkgraaf, M.G.W.; Gerlag, D.M.; van Eck-Smit, B.L.F.; Tak, P.P. Bone Mineral Density in Rheumatoid Arthritis Patients 1 Year after Adalimumab Therapy: Arrest of Bone Loss. Ann. Rheum. Dis. 2009, 68, 373-376. [CrossRef] 
65. Eekman, D.A.; Vis, M.; Bultink, I.E.M.; Kuik, D.J.; Voskuyl, A.E.; Dijkmans, B.A.C.; Lems, W.F. Stable Bone Mineral Density in Lumbar Spine and Hip in Contrast to Bone Loss in the Hands during Long-Term Treatment with Infliximab in Patients with Rheumatoid Arthritis. Ann. Rheum. Dis. 2011, 70, 389-390. [CrossRef] [PubMed]

66. Lange, U.; Teichmann, J.; Müller-Ladner, U.; Strunk, J. Increase in Bone Mineral Density of Patients with Rheumatoid Arthritis Treated with Anti-TNF-Alpha Antibody: A Prospective Open-Label Pilot Study. Rheumatol. Oxf. Engl. 2005, 44, 1546-1548. [CrossRef]

Publisher's Note: MDPI stays neutral with regard to jurisdictional claims in published maps and institutional affiliations.

(C) 2020 by the authors. Licensee MDPI, Basel, Switzerland. This article is an open access article distributed under the terms and conditions of the Creative Commons Attribution (CC BY) license (http://creativecommons.org/licenses/by/4.0/). 